\title{
Notas sobre la cocina de autor(a)
}

\author{
JULIETA FLORES JURADO \\ Universidad Nacional Autónoma de México
}

\section{Resumen}

En 1969, en su conferencia “¿Qué es un autor?”, Michel Foucault señaló que la función autor no es "universal y constante", sino que opera en un conjunto diverso y cambiante de discursos y prácticas. Si entendemos la autoría, siguiendo a Foucault, como un momento privilegiado de individuación, considero que es posible identificar una figura de autor emergente en la gastronomía, que se basa tanto en la definición del cocinero como una figura con autoridad y prestigio en el campo cultural y en la atribución de una firma de autor a su comida, como en la exclusión de sujetos a los que históricamente se les ha negado la posibilidad de ocupar un lugar de autor. A partir de cuestionar expresiones como "escritor gastronómico" y "cocina de autor", reflexionaré sobre la construcción de la gastronomía como campo cultural, con un énfasis especial en la participación de las mujeres gastrónomas, y en las estrategias que han empleado para definirse como autoras en un sistema que tradicionalmente ha ignorado las expresiones del apetito y la creatividad femeninas.

Palabras clave: autor, cocina de autor, gastronomía, escritura gastronómica, estudios alimentarios feministas

\begin{abstract}
In 1969, in his lecture "What is an Author?", Michel Foucault argued that the author function is not "universal and constant", but instead operates within a diverse and mutable range of disciplines and discourses. If authorship can be understood, according to Foucault, as a privileged moment of individualization, it is possible to identify an emerging authorial figure in gastronomy that is based, on the one hand, on defining the cook as a figure of authority and prestige in the cultural field whose food can bear the signature of an author, and on the other, on the exclusion of subjects who have been historically discouraged and prevented from occupying an authorial position. Calling into question concepts such as "food writer" and "cocina de autor" (author's cuisine), I propose a brief account of the invention of gastronomy as a literary field, with a special emphasis on the role of women gastronomes, and the strategies they have deployed to define themselves as authors within a system that traditionally has ignored the expression of female appetite and creativity.
\end{abstract}

Keywords: author, cocina de autor, gastronomy, food writing, feminist food studies 
the dish had been invented by the chef of the very café in which they were dining, a person known all over Paris as the greatest culinary genius of the age, and-most surprisingly-a woman!

-Isak Dinesen [Karen Blixen], "Babette's Feast”

n las últimas décadas, la expresión "cocina de autor" se ha empleado para
describir un tipo de comida que encarna el estilo y la creatividad indivi-
duales de un cocinero, que reinterpreta recetas y técnicas tradicionales de maneras innovadoras, y que comúnmente se sirve en un formato llamado "menú degustación": una secuencia de platillos cuyo orden y lógica interna no dependen de la elección de los comensales, sino de los ingredientes de temporada y especialmente de la imaginación del chef. Se trata de un movimiento que concede un gran valor a la originalidad: los cocineros que se identifican con la cocina de autor desarrollan recetas únicas que reflejan un proyecto personal. Parte importante de la cocina de autor es la presencia pública del cocinero fuera de su cocina: se trata de una figura mediagénica, que ya no sólo es autor o coautor de libros de cocina, sino también presentador de programas de televisión, jurado en concursos, activista o sujeto de biografías y documentales. Algunos cocineros, como René Redzepi, han publicado facsímiles de sus diarios o presentado en salas de museos los bocetos de sus creaciones comestibles. Esta transformación es el resultado de un largo proceso de construcción de la gastronomía como un campo artístico, y de la labor de cocineros y escritores que han defendido la cocina como una forma de arte. En suma, la expresión "cocina de autor" designa una nueva forma de concebir al cocinero como creador, genio y artista, en contraste con un saber culinario colectivo y anónimo.

En este trabajo, propongo leer la cocina de autor en diálogo con algunos de los comentarios de Michel Foucault en su influyente conferencia de 1969, “¿Qué es un autor?”. En ella, Foucault señala que la función autor no es constante en todos los discursos ni en todas las épocas; los discursos cuyo modo de circulación no involucraba la función autor pueden adquirirla en un momento específico de su existencia, al coincidir diferentes factores que permitan lo que Foucault llama un "momento fuerte de individuación" (2009: 115). 
En las primeras décadas del siglo XXI, la gastronomía parecería estar viviendo este momento: ser un creador gastronómico hoy implica autonomía, autoridad, originalidad e iniciativa, cualidades asociadas a la autoría (Pease, 1995: 105). Los cocineros más celebrados ya no dependen de los dueños de los hoteles o de la aristocracia; ahora son dueños o socios de sus propios restaurantes. Su comida ya no se sirve directamente en fuentes o cacerolas; al construir cuidadosamente porciones individuales, el cocinero traza una intención específica en cada plato como si fuera una página en blanco. Estas transformaciones son una oportunidad para reflexionar sobre la existencia de una noción de autor en la gastronomía, y para considerar algunas de las estrategias que las cocineras han empleado para negociar con un campo cultural que sistemáticamente ha rechazado las expresiones del apetito femenino y la integración de las mujeres a las cocinas profesionales.

Vuelvo a “¿Qué es un autor?”: Foucault señala que los relatos sobre las vidas de los héroes han dado paso a un nuevo tipo de vida "narrable", la vida del autor (2009: 115). Quizá podríamos pensar que en estas biografías no opera una sustitución, sino un desplazamiento más sutil: los autores modernos se han heroizado. Por lo tanto, al situarse el cocinero como una figura autoral, su vida puede narrarse como una vida heroica. Un ejemplo reciente de estas conexiones entre autoría y autoridad, cocinero y héroe, puede encontrarse en el primer episodio de la serie de documentales Chef's Table. El protagonista es Massimo Bottura, chef y propietario de Osteria Francescana, un restaurante en Módena reconocido como uno de los diez mejores del mundo por diferentes instituciones gastronómicas. En mayo de 2012, un terremoto dañó más de trescientas mil ruedas de queso Parmiggiano Reggiano que reposaban en bodegas cerca de la ciudad de Módena. El desastre podría haber significado la quiebra para miles de productores de queso y una seria amenaza a uno de los productos más representativos de la cocina de Emilia-Romaña. En respuesta a la petición de ayuda del presidente del consorcio del Parmiggiano Reggiano, Massimo Bottura ideó una receta de risotto con queso y pimienta, cacio e pepe, e invitó a otros chefs a servirla en sus restaurantes en solidaridad con los productores de queso modeneses. Poco tiempo después, todo el queso se había vendido. El documental 
presenta al chef como un héroe que usó su talento e influencia para salvar a los productores de queso de su ciudad natal.

El trabajo de Bottura (Figura 1) es un ejemplo claro de la transformación del cocinero en autor: la cocina que sirve en Osteria Francescana se ha nutrido tanto de las recetas tradicionales como del arte de vanguardia, y su postura autoral va mucho más allá de la labor de dirigir un restaurante. Sin embargo, como sucede con la autoría literaria, la autoría gastronómica está diseñada para un autor hombre. En un artículo, la periodista Jane Kramer (2013) se refiere a Bottura como

one of a small, far-flung brotherhood of exceptionally gifted and inventive chefs who have deconstructed, distilled, concentrated, and, with uncommon respect, reconstructed the flavors of their own traditional cuisines. They are in constant touch. They text, they tweet, they call. They travel across the planet to share their ideas and secrets and techniques [...] They convene in August for the MAD weekend — the Noma chef René Redzepis annual gathering of the tribe in Copenhagen. They fish and hunt and forage and cook together at wilderness outings like Cook It Raw, gastronomy's extreme sport.

Estos "deportes extremos" cumplen una función muy específica en la configuración de la gastronomía como campo artístico: contrarrestan la asociación naturalizada entre comida y feminidades. Como demuestran Deborah Harris y Patti Giuffre (2015), en las cocinas profesionales existe una condición de masculinidad precaria: el arte del cocinero está constantemente amenazado por sus conexiones con el trabajo doméstico, feminizado y devaluado. Para elevar la cocina a la categoría de arte, el campo gastronómico busca distanciarse enfáticamente de la cocina cotidiana y del espacio doméstico, y por ende del trabajo feminizado de cuidados que se asocia con este contexto:

For chefs, [their] masculine, professional activity is always at risk of comparison to the unpaid, nonprofessional cooking of women in the home. Feminization threat leads to a sense of insecure and unstable masculinity amongst men in jobs requiring them to perform female-coded tasks 
Figura 1

La cocina de autor de Massimo Bottura

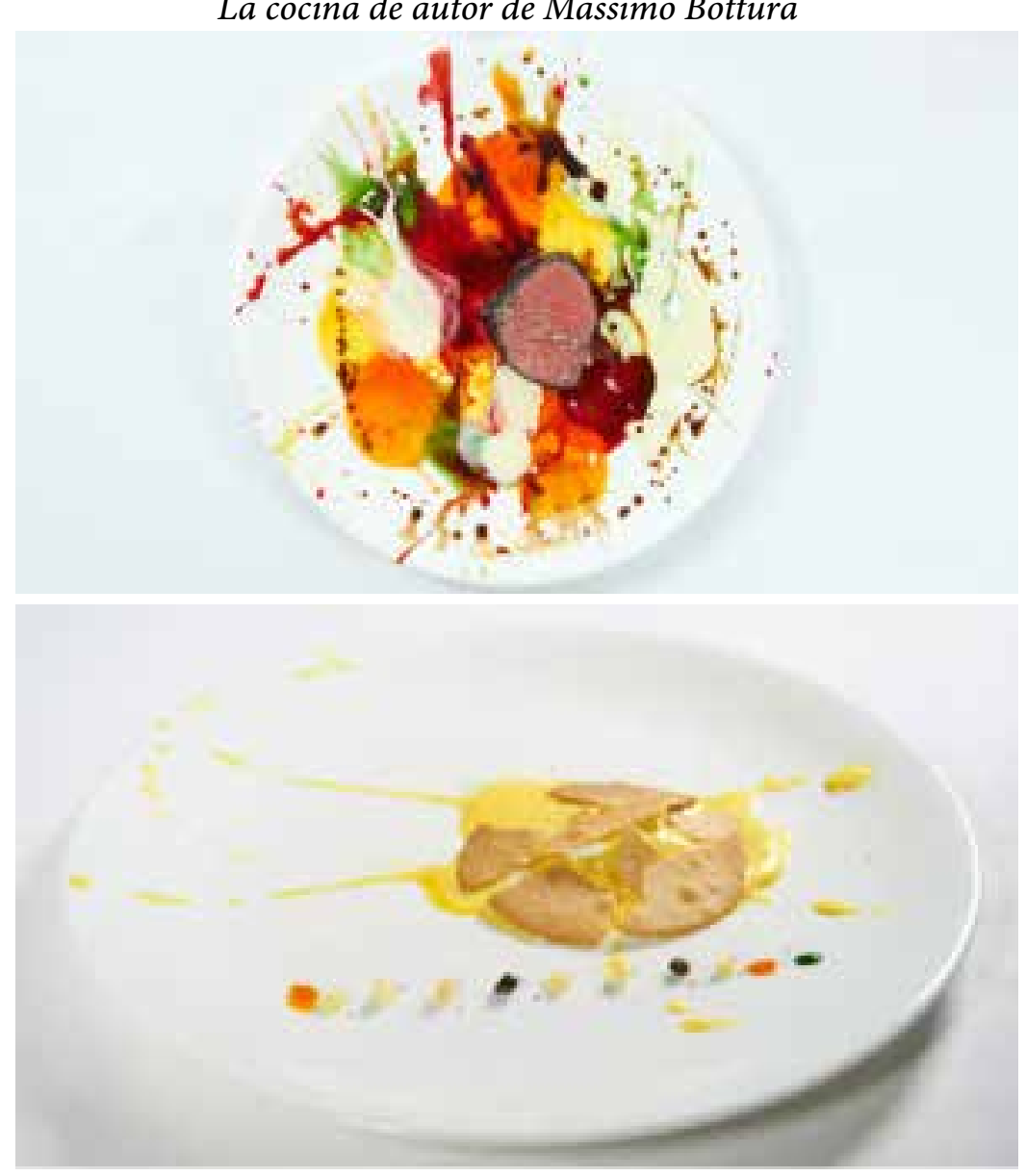

Nota: Beautiful psychedelic spin-painted veal, not flame grilled (arriba) y Oops! I dropped the lemon tart (abajo). Fotografías de Gideon Hart.

-a term we refer to as precarious masculinity. To neutralize feminization threat and prevent men workers from experiencing precarious masculinity, several actions can be taken, including emphasizing the masculine nature of their work (e. g., focusing on the military background of their occupation) while de-emphasizing skills or attributes that could be considered feminine (e. g., caring for others). (Harris y Giuffre: 2015: 9)

Los efectos de esta polarización de la cocina pueden observarse en las formas parciales de juzgar el trabajo de las cocineras profesionales: su comida se describe como "maternal", "instintiva", como si esto indicara únicamente una exten- 
sión de sus inclinaciones "naturales" y no un verdadero mérito artístico. Otro aspecto de la cita de Kramer que habla sobre la exclusión de las mujeres de las posiciones de poder en el campo gastronómico es el uso de la palabra brotherhood. Hay un árbol genealógico de los grandes cocineros de la historia, y en esta familia de artistas es casi imposible encontrar el nombre de una mujer antes de la segunda mitad del siglo XX. En 1895, la ensayista y crítica de arte Elizabeth Robins Pennell lamentaba el hecho de que "the kitchen still waits its Sappho" (1900: 6).

La exclusión de las mujeres de la escritura gastronómica aparece ya en los textos que inauguran este género. En 1825, Jean Anthelme Brillat-Savarin definió la gastronomía como "el conocimiento razonado de todo lo que concierne a la alimentación del hombre" (2009: 106, las cursivas son mías). Hay una figura femenina en La fisiología del gusto, Gasterea, a quien el autor llama "la décima musa", pero Brillat-Savarin, como otros escritores de la época, no contempla la posibilidad de una mujer gastrónoma, pues su disciplina se encontraba determinada por "ideological constructs that align[ed] a women's appetite for food, for public voice, and for economic, political, or social power with greed and moral corruption" (McLean, 2013: 251). Por otra parte, hablar de comida es acercarse frecuentemente, advierte Brad Kessler, a temas como "carnality, appetite, desire [...] The perishability and baseness of the body. And, of course, sex [...] So it's not surprising that some authors are uncomfortable with the whole affair of eating" (2005: 157-158). Estos temas serían incluso más incómodos cuando fuera una autora quien sostuviera la pluma.

Por otra parte, la trama de aventuras y hazañas heroicas, como argumenta Joanna Russ (1972), presupone a un personaje masculino como protagonista. Si los mitos o tramas más comunes dificultan pensar en un personaje femenino como una figura heroica, pues "una mujer no narra su propia historia, ni es personaje del enunciado, no suele fungir como personaje focal y la estructura apelativa del texto no da cabida a una lectora implícita" (Golubov, 2012: 36-37), postular a una cocinera como autora y como heroína conlleva un doble obstáculo por las evidentes conexiones de la cocina con la corporalidad, aparentemente contraria a la neutralidad, racionalidad y universalidad que distinguen a la noción occidental de la autoría. 
Quizá los gastrónomos del siglo XIX no habrían anticipado que entre sus discípulos se encontrarían varias mujeres de buen apetito a quienes Alice McLean, siguiendo a Paul Schmidt, ha llamado "savarinists" (2012: 6), entre ellas las escritoras Elizabeth David, Alice B. Toklas y M. F. K. Fisher -esta última incluso tradujo la obra de Brillat-Savarin al inglés.

Quiero concentrarme ahora en cómo las autobiografías escritas por mujeres cocineras y gastrónomas plantean una alternativa a la oposición cuerpo-intelecto al demostrar la posibilidad de narrarse y contar una historia de crecimiento intelectual a través de la comida. La autobiografía gastronómica, también llamada food memoir o incluso foodoir (Gilbert, 2014: 141), no es un género del siglo XXI. Las mujeres han articulado narrativas personales a través o a la par de sus recetas durante muchos siglos, pero la reciente visibilidad de esta práctica se debe en gran medida a las intervenciones feministas que, como adelantaba Lillian Robinson en su discusión sobre feminismo y canon literario, han cuestionado los criterios estéticos bajo los que se construye el canon y han permitido el redescubrimiento de géneros "menores", como cartas, diarios, autobiografías, novelas rosas y libros de cocina.

Cabe aclarar que los textos gastronómicos no fueron inmediatamente bienvenidos por la crítica literaria feminista. La cocina se leyó en un principio como un sitio de confinamiento e invisibilización que reproducía la desigualdad. En su primera etapa, los estudios alimentarios feministas se enfocaron en analizar los diferentes mensajes que las mujeres o los personajes femeninos transmitían por medio del rechazo a la comida. Warren Belasco afirma que para la academia ha sido difícil dejar atrás la conexión entre comida y opresión (2008: 3). Sin embargo, otras investigadoras reconocen que, aunque la cocina puede ser efectivamente un sitio que refleja y reproduce la subordinación de las mujeres y la devaluación de su trabajo creativo, para muchas mujeres la cocina también ha significado "a vehicle for artistic expression, a source of sensual pleasure, an opportunity for resistance" (Avakian et al., cit. en McLean, 2013: 252). Es difícil, entonces, aproximarse al significado de la comida y la cocina en la vida de las mujeres desde un solo punto de vista. Elegir o rechazar cierta comida es siempre un acto significativo, y el hecho de que una mujer encuentre en la cocina una forma de expresión no debe ser descalificado como trivial. 
Un ejemplo temprano de la recuperación de la cocina como un espacio de creatividad y empoderamiento, que además presenta a una cocinera como figura heroica, es el libro de Irma Rombauer The Joy of Cooking, publicado por primera vez en 1931 (Figura 2). Aunque se trata de un libro de recetas, textos que parecerían ser primordialmente técnicos o funcionales, The Joy of Cooking sentó algunas de las bases que permitirían pensar la vida de las cocineras como una narrativa literaria. En primer lugar, el título señala un cambio de reglas para las lectoras. Hablar de la cocina como un placer, y no como un deber, era un gesto osado en el contexto en el que escribía Rombauer. La portada del libro, que Rombauer diseñó en colaboración con su hija Marion, muestra a una mujer armada con algunos utensilios domésticos que se enfrenta a un dragón. Esta mujer, como indica su halo verde y azul, es Santa Marta de Betania, patrona de las cocineras y de las amas de casa. El dragón enemigo representa el lado tedioso de la cocina.

Figura 2

Facsímil de la edición de 1931 de The Joy of Cooking

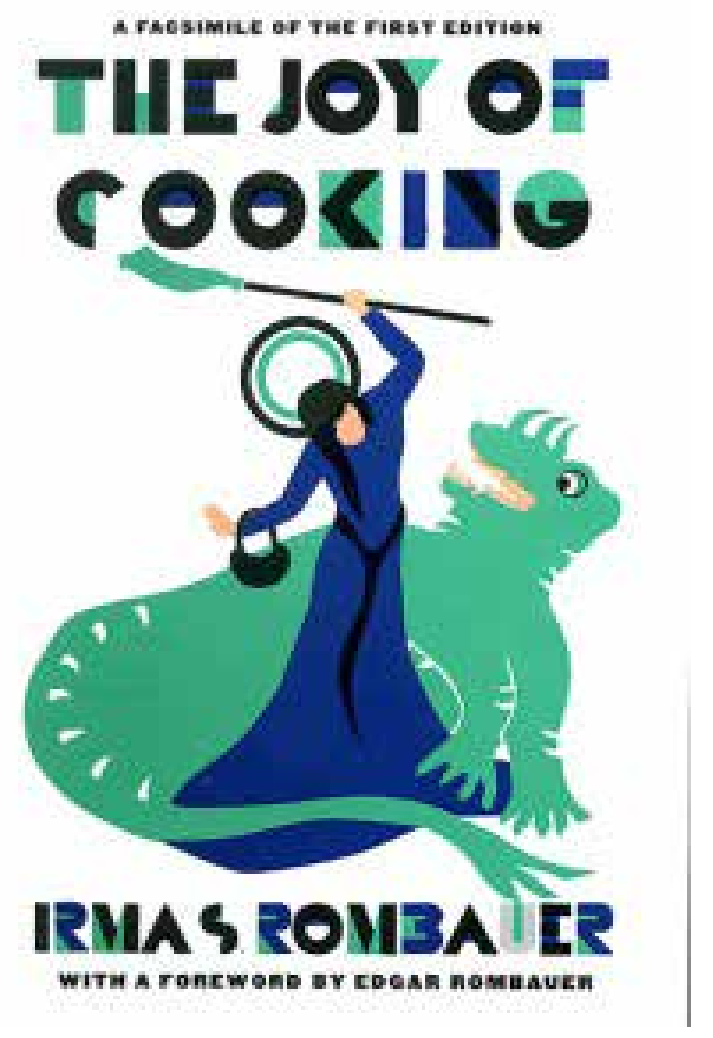


La autora encuentra un paralelo entre Santa Marta y la lectora moderna, quien se enfrenta, explica Rombauer en el prólogo, a "tasks almost as diversified as St. Marta's, and cooking is not the least of the problems she must meet with intelligence and understanding" (cit. en Ferguson, 2014: 91). Pero hay algo más: la presentación de Santa Marta como una figura heroica niega la idea de la cocina como una pesada obligación, y la convierte en una aventura. Santa Marta aparece como una versión femenina de San Jorge, pero ella lucha una cruzada por la reivindicación del placer de la cocina y la creatividad gastronómica femenina. La cocinera ha sido heroizada y canonizada. ${ }^{1}$

El trabajo de las historiadoras del arte feministas en las décadas de 1970 y 1980; la teoría literaria que comenzó a reflexionar sobre el cuerpo de las autoras y su lugar en el canon; el performance, que hizo del cuerpo de la artista la base de la obra de arte, y la recuperación por parte de las artistas de técnicas consideradas menores, como el bordado o el patchwork, han sido otros factores que han hecho posible pensar en una cocinera como figura autoral. Si bien libros como The Joy of Cooking han sido la base de una nueva historia sobre las relaciones positivas que las mujeres pueden establecer con la comida, y la cocina se ha representado como el estudio de una artista - en el cuento de Isak Dinesen "Babette's Feast" y en su adaptación al cine, por ejemplo-, también es cierto que en el repertorio de tramas para una gastrónoma y de posturas autorales que puede ocupar una cocinera aún persisten limitaciones.

Un estudio sobre las personas (en el sentido de personajes o máscaras) actuadas por cuarenta y cuatro celebrity chefs concluye que un gran porcentaje de libros y programas de cocina contribuye a perpetuar normas de género tradicionales e idealizadas (Johnston, Rodney y Chong, 2014: 5). Estos textos culturales suelen presentar a la mayoría de las cocineras en el espacio doméstico, sin uniformes de chef, y a los cocineros vestidos con filipinas, trabajando en restaurantes y asociados a un espacio en el que "authority and legitimacy is derived from the creative inspiration of an artistic genius, as well as from knowledge

1 Agradezco a Aina Pérez Fontdevila y a mis compañeros del seminario La autoría en escena por escuchar y comentar mi análisis de la portada de The Joy of Cooking. 
and power gained through formal training and earned credentials" (Johnston, Rodney y Chong, 2014: 13).

Como en las tramas arquetípicas que deconstruye Russ, las personas culturalmente disponibles para las cocineras refieren a formas de legitimidad y autoridad que "echo the historic binary between the less-valued female home-cook [...] and the publicly celebrated male chef" (Johnston, Rodney y Chong, 2014: 18). Harris y Giuffre coinciden en este punto: al entrevistar a treinta y tres mujeres chefs, las sociólogas identificaron tres estilos de presentación y ejercicio de la autoridad empleados por las cocineras: "bitches", "girly girls" o "moms/ big sisters" (2015: 130). Evidentemente, las tres presentaciones son sumamente reductivas y estereotípicas. Entre estas tres formas de autoridad, la figura de "mom/big sister" era la alternativa preferida por las chefs; sin embargo, Harris y Giuffre desconfían de esta imagen porque también se basa en una concepción esencialista del sexo-género: "anything that supports the gender binary ends up promoting gender inequality because society always devalues the feminine" (2015: 159).

Un texto que describe la negociación de una cocinera con este sistema de desventajas es la autobiografía Blood, Bones \& Butter de Gabrielle Hamilton, chef ejecutiva y propietaria del restaurante Prune en Nueva York y una de las cocineras-autoras más populares en los últimos años. En este relato, el cuerpo femenino nuevamente está en el centro del debate. Hamilton, quien ha sido descrita por otras cocineras como "the Patti Smith of [our] industry" (Druckman, 2012: 233), narra su formación gastronómica a partir de tres símbolos, los primeros dos fuertemente ligados al cuerpo de la cocinera: la sangre, los huesos y la mantequilla. El relato hace un énfasis especial en la búsqueda de predecesoras, pero para la generación de Hamilton no es posible encontrar a estas predecesoras en los restaurantes premiados con estrellas Michelin. Las figuras maternas que forman a Hamilton son Madeleine, su madre biológica; Misty, la chef de una sencilla compañía de banquetes, y Alda, su suegra italiana.

La autobiografía tiene todos los ingredientes para leerse como un ejemplo sumamente interesante de la intersección entre feminidades, comida y creación artística: una protagonista independiente que desafía el sexismo de las cocinas profesionales; la conexión con figuras maternas alternativas al canon gastronó- 
mico; un restaurante que sirve un menú autobiográfico; la legitimidad como creadora a partir de credenciales poco convencionales, como el simple hecho de tener hambre, y la defensa del apetito femenino ante la propagación de un ideal de delgadez imposible. Sin embargo, la autobiografía, que incorpora elementos de una narrativa de formación o Bildungsroman, no logra plenamente crear una imagen de la cocinera como una subjetividad completa. Hamilton frecuentemente tiene que decidir entre actuar como mujer o actuar como chef, porque estos dos roles parecerían difíciles de reconciliar. Hamilton describe así su participación en un congreso de mujeres chefs donde los organizadores exigen a las ponentes usar una filipina:

I couldn't decide if I wanted to emphasize my femaleness or my chef-ness. If I were emphasizing my chef-ness, I would have worn the jacket. But if I was emphasizing my femaleness and my confidence in my equal abilities and talents, I wouldn't want to wear the jacket. I would want to say, sartorially, that I am so confident and secure in my role that I don't need the costume to prop me up (2011: 209).

La organización del congreso coloca así a las cocineras en una escenografía autoral que indica, desafortunadamente, que a pesar de la conexión naturalizada entre mujeres y cocina, se necesita de un "disfraz" para que los espectadores perciban a estas mujeres como chefs (en la acepción literal de la palabra, como autoridades). Estas tensiones en la imagen pública de la cocinera hablan sobre la necesidad de nuevas representaciones que construyan subjetividades en las que feminidad y gastronomía, autoría y maternidad, y cuerpo y expresión artística puedan existir simultáneamente, como parte de una identidad tal vez múltiple o contradictoria, pero no por ello mutilada o incompleta. El Bildungsroman femenino o Frauenroman, en especial cuando involucra a una cocinera como narradora y protagonista, por la historia paradójica de esta figura, puede ser uno de los espacios donde comiencen estas exploraciones.

En las décadas posteriores a que Foucault identificara la función autor, algunas críticas feministas argumentaron que había que descartar también esta noción, eliminar toda idea de presencia autoral para liberar verdaderamente la lectura. ¿Para qué revalorar al autor si su figura remitía justo al sistema que se 
buscaba criticar? (Walker, 1990: 555). Otras lectoras, como Nancy K. Miller, pensaban que "the removal of the Author has not so much made room for a revision of the concept of authorship as it has, through a variety of rhetorical moves, repressed and inhibited discussion of any writing identity in favor of the (new) monolith of anonymous textuality" (cit. en Walker, 1990: 560). Al evaluar estas dos posturas, Cheryl Walker concluye que "The loss of the writer runs us the risk of losing many stories important to our history. Radical freedom [...] may in the end leave us without the tools necessary to consider the way biography and fiction are in dialogue with one another and provide a critique of patriarchy as well as, in some cases, models of resistance" (1990: 560).

Considero que una de las "many stories" que Walker revalora es precisamente la historia de las cocineras y su posición ambivalente entre la feminidad esencializada de la cocina doméstica y el difícil acceso a la cocina de autor. El estudio de su resistencia y negociación al interior del campo gastronómico es un posible camino hacia una consideración crítica de conceptos como canon y autoría.

\section{Referencias bibliográficas}

Belasco, Warren (2008): Food: The Key Concepts. Nueva York: Berg.

Brillat-Savarin, Jean Anthelme (2009): The Physiology of Taste or Meditations on Transcendental Gastronomy (trad. M. F. K. Fisher). Nueva York: Everyman's Library / Alfred A. Knopf.

Dinesen, Isak [Karen Blixen] (2011): Babette's Feast. Londres: Penguin.

Druckman, Charlotte (2012): Shirt Steak. Women Chefs on Standing the Heat and Staying in the Kitchen. San Francisco: Chronicle Books.

Ferguson, Priscilla Parkhurst (2014): Word of Mouth: What We Talk About When We Talk About Food. Oakland / Londres: University of California Press. 
Foucault, Michel (2009): “¿Qué es un autor?”. En María Stoopen Galán (coord.), Sujeto y relato. Antología de textos teóricos. México: Universidad Nacional Autónoma de México, Facultad de Filosofía y Letras.

GILBERT, Sandra M. (2014): The Culinary Imagination: From Myth to Modernity. Londres / Nueva York: W. W. Norton.

Golubov, Nattie (2012): La crítica literaria feminista. Una introducción práctica. México: Universidad Nacional Autónoma de México.

Hamilton, Gabrielle (2011): Blood, Bones \& Butter. The Inadvertent Education of a Reluctant Chef. Nueva York: Random House.

Harris, Deborah A.; y Giuffre, Patti (2105): Taking the Heat: Women Che$f s$ and Gender Inequality in the Professional Kitchen. New Brunswick, Nueva Jersey: Rutgers University Press.

Johnston, Josée; Rodney, Alexandra; y Chong, Phillipa [2014]: "Making Change in the Kitchen? A Study of Celebrity Cookbooks, Culinary Personas, and Inequality”. En Poetics. Vol. 47, diciembre, pp. 1-22.

Kessler, Brad (2005): “Towards a Gastronomic Theory of Literature”. En Kenyon Review. New Series. Vol. 27, núm. 2, primavera, pp. 148-165.

Kramer, Jane (2013): “Post-Modena”. En The New Yorker. [Consulta: 28 de octubre de 2015].

McLean, Alice (2012): Aesthetic Pleasure in Twentieth Century Women's Food Writing: The Innovative Appetites of M. F. K. Fisher, Alice B. Toklas and Elizabeth David. Nueva York: Routledge.

McLean, Alice (2013): “The Intersection of Gender and Food Studies”. En Ken Albala (ed.), Routledge International Handbook of Food Studies. Nueva York: Routledge.

Pease, Donald E. (1995): "Author”. En Frank Lentricchia y Thomas McLaughlin (eds.), Critical Terms for Literary Study. Chicago: University of Chicago Press, pp. 105-17. 
Pennell, Elizabeth Robins (1900): The Feasts of Autolycus. The Diary of a Greedy Woman. Chicago / Nueva York: The Saalfield Publishing Company

Robinson, Lillian S. (1983): “Treason Our Text: Feminist Challenges to the Literary Canon”. En Tulsa Studies in Women's Literature. Vol. 2, núm. 1, primavera, pp. 83-98.

Russ, Joanna (1972): "What Can A Heroine Do? or Why Women Can't Write?”. En Susan Koppelman Cornillon (ed.), Images of Women in Fiction. Feminist Perspectives. Ohio: Bowling Green University Popular Press, pp. 3-20.

WALKer, Cheryl (1990): “Feminist Literary Criticism and the Author". En Critical Inquiry. Vol. 16, núm. 3, primavera, pp. 551-571. 